Linköping Studies in Science and Technology

Thesis No. 1734

\title{
Generalised Ramsey numbers and Bruhat order on involutions
}

\author{
Mikael Hansson
}

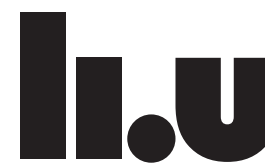

LINKÖPING UNIVERSITY

Department of Mathematics

Linköping University

SE-581 83 Linköping, Sweden

Linköping 2015 
Generalised Ramsey numbers and Bruhat order on involutions Mikael Hansson

Department of Mathematics

Linköping University

SE-581 83 Linköping, Sweden

mikael.hansson@liu.se

Linköping Studies in Science and Technology, Thesis No. 1734

ISSN 0280-7971

ISBN 978-91-7685-892-9

Copyright (C) 2015 Mikael Hansson

Printed by LiU-Tryck, Linköping, Sweden, 2015 


\section{Abstract}

This thesis consists of two papers within two different areas of combinatorics.

Ramsey theory is a classic topic in graph theory, and Paper A deals with two of its most fundamental problems: to compute Ramsey numbers and to characterise critical graphs. More precisely, we study generalised Ramsey numbers for two sets $\Gamma_{1}$ and $\Gamma_{2}$ of cycles. We determine, in particular, all generalised Ramsey numbers $R\left(\Gamma_{1}, \Gamma_{2}\right)$ such that $\Gamma_{1}$ or $\Gamma_{2}$ contains a cycle of length at most 6 , or the shortest cycle in each set is even. This generalises previous results of Erdôs, Faudree, Rosta, Rousseau, and Schelp. Furthermore, we give a conjecture for the general case. We also characterise many $\left(\Gamma_{1}, \Gamma_{2}\right)$ critical graphs. As special cases, we obtain complete characterisations of all $\left(C_{n}, C_{3}\right)$-critical graphs for $n \geq 5$, and all $\left(C_{n}, C_{5}\right)$-critical graphs for $n \geq 6$.

In Paper B, we study the combinatorics of certain partially ordered sets. These posets are unions of conjugacy classes of involutions in the symmetric group $S_{n}$, with the order induced by the Bruhat order on $S_{n}$. We obtain a complete characterisation of the posets that are graded. In particular, we prove that the set of involutions with exactly one fixed point is graded, which settles a conjecture of Hultman in the affirmative. When the posets are graded, we give their rank functions. We also give a short, new proof of the EL-shellability of the set of fixed-point-free involutions, recently proved by Can, Cherniavsky, and Twelbeck. 



\section{Populärvetenskaplig sammanfattning}

Den här uppsatsen består av två artiklar inom olika områden av kombinatorik.

Ramseyteori, som är temat för den första artikeln, är ett klassiskt ämne inom grafteori. En graf består av ett antal hörn och ett antal kanter som förbinder vissa av hörnen. Den kompletta grafen har en kant mellan varje par av hörn, och en röd-blå graf är en komplett graf med varje kant färgad antingen röd eller blå. Det generaliserade ramseytalet $R\left(\mathcal{G}_{1}, \mathcal{G}_{2}\right)$ är det minsta positiva heltalet $n$, sådant att varje röd-blå graf på $n$ hörn innehåller en röd kopia av en graf från mängden $\mathcal{G}_{1}$ eller en blå kopia av en graf från mängden $\mathcal{G}_{2}$. Ramseytalet beskriver alltså hur många hörn en röd-blå graf måste ha för att den garanterat ska innehålla någon av vissa specificerade delgrafer. Startpunkten för ramseyteori var Frank Ramseys bevis 1930 av att alla ramseytal existerar, dvs. att om bara grafen har tillräckligt många hörn, så kommer den alltid att innehålla någon av de specificerade delgraferna. Ämnet tog fart på allvar på 1950-talet och idag är det ett av de mest aktiva fälten inom grafteori.

Vi studerar generaliserade ramseytal $R\left(\mathcal{G}_{1}, \mathcal{G}_{2}\right)$ där mängderna $\mathcal{G}_{1}$ och $\mathcal{G}_{2}$ består av cykler (om man ritar upp grafen så ser den ut som en cykel). Detta problem löstes på 1970-talet då mängderna endast innehåller en cyckel var, men det allmänna problemet har bara delvis studerats tidigare. Vi bestämmer alla sådana generaliserade ramseytal där $\mathcal{G}_{1}$ eller $\mathcal{G}_{2}$ (eventuellt båda) innehåller en cykel med högst 6 hörn.

Förutom att beräkna ramseytal, försöker matematiker att beskriva de grafer som inte innehåller någon av de specificerade delgraferna. Framför allt försöker man att karaktärisera de $\left(\mathcal{G}_{1}, \mathcal{G}_{2}\right)$-kritiska graferna, dvs. ge en beskrivning av precis de grafer på $R\left(\mathcal{G}_{1}, \mathcal{G}_{2}\right)-1$ hörn som varken innehåller en röd delgraf från $\mathcal{G}_{1}$ eller en blå delgraf från $\mathcal{G}_{2}$. Vi karaktäriserar alla $\left(C_{n}, C_{3}\right)$-kritiska grafer för $n \geq 5$, och alla $\left(C_{n}, C_{5}\right)$-kritiska grafer för $n \geq 6$.

I den andra artikeln studerar vi kombinatoriken hos vissa partialordnade 
mängder (eller pomängder). Det är mängder där en del element är större än andra, men det finns i allmänhet också element som inte är jämförbara med varandra (inget av elementen är större än det andra). Den symmetriska gruppen $S_{n}$ består av alla permutationer av talen $1,2, \ldots, n$, dvs. alla uppräkningar av talen, till exempel 3,2,1,5,4 eller 2,3,1,5,4. Permutationerna kan ordnas med den så kallade Bruhatordningen, som har viktiga kopplingar till algebra och geometri. På så vis blir $S_{n}$ en pomängd.

En involution i $S_{n}$ är en permutation som fås genom att bara talen inom vissa par byter plats med varandra. Till exempel är 3,2,1,5,4 en involution ( 1 byter plats med 3 och 4 byter plats med 5), men 2,3,1,5, 4 är det inte. Det är naturligt att betrakta mängden $I_{n}$ av alla involutioner som en pomängd, där ordningen bestäms av Bruhatordningen på $S_{n}$. Man kan också betrakta vissa delmängder av $I_{n}$ som pomängder, till exempel $F_{n}^{0}$, de involutioner som inte har några fixpunkter (ovan är 2 den enda fixpunkten, ty talet 2 står på plats 2). Vi karaktäriserar de pomängder som är graderade. Det är en viktig egenskap som en pomängd kan ha, och betyder ungefär att elementen i pomängden kan delas in i olika "nivåer". Vi bestämmer också rangfunktionen av de graderade pomängderna. Den beskriver vilken "nivå" varje element tillhör. Vi ger också ett kort, nytt bevis av att $F_{n}^{0}$ är EL-skalbar. Det betyder att pomängden kan "märkas" på ett sätt som säger något om dess topologi. 


\section{Acknowledgements}

First and foremost, I want to thank my supervisor, Axel Hultman, for his patience, support, and many comments on earlier versions of the included papers. I also want to thank my second supervisor, Jan Snellman, and my MSc thesis advisor, Jörgen Backelin. Thanks go to all my colleagues and fellow PhD students at the Department of Mathematics. Finally, I want to thank my family. 



\section{Contents}

I Introduction 1

1 Ramsey theory and Bruhat order 3

1.1 Ramsey theory ................... . . 3

1.2 The Bruhat order and poset topology . . . . . . . . 5

1.2 .1 Posets . . . . . . . . . . . . . . . . . . 5

1.2 .2 Coxeter groups . . . . . . . . . . . . . . . . 6

1.2 .3 Shellability . . . . . . . . . . . . . . . 8

1.2.4 The Bruhat order on involutions . . . . . . . . . . 9

2 Summary of papers $\quad 11$

Paper A: Generalised Ramsey numbers for two sets of cycles . 11

Paper B: The Bruhat order on conjugation-invariant sets of involutions in the symmetric group . . . . . . . . . . . 11

$\begin{array}{ll}\text { Bibliography } & 14\end{array}$

$\begin{array}{ll}\text { II Papers } & 15\end{array}$ 



\section{Part I}

\section{Introduction}





\section{Chapter 1}

\section{Ramsey theory and Bruhat order}

For a non-negative integer $n$, let $[n]=\{1,2, \ldots, n\}$ (so $[0]=\emptyset$ ), and for integers $a \leq b$, let $[a, b]=\{a, a+1, \ldots, b\}$.

\subsection{Ramsey theory}

Ramsey theory is a classic topic in graph theory. In this section, we give its basic definitions and some of its classic results; we also mention a recent result that is of interest to us.

We consider only non-empty graphs that are finite, simple, and undirected. Also, $\left(G_{1}, G_{2}\right)$ and $\left(\mathcal{G}_{1}, \mathcal{G}_{2}\right)$ denote a pair of graphs and a pair of non-empty sets of graphs, respectively. By a red-blue graph we shall mean a complete graph with each edge coloured either red or blue. Red will always be the first colour and blue will always be the second.

We have the following definition:

Definition 1.1.1. The Ramsey number $R\left(G_{1}, G_{2}\right)$ is the least positive integer $n$, such that each red-blue graph on $n$ vertices contains $G_{1}$ as a red subgraph or $G_{2}$ as a blue subgraph; let $R(i, j)=R\left(K_{i}, K_{j}\right)$.

It was proved by Ramsey [13] in 1930 that $R\left(G_{1}, G_{2}\right)$ always exists. Clearly, $R\left(G_{1}, G_{2}\right)=R\left(G_{2}, G_{1}\right), R\left(G, K_{1}\right)=1$, and $R\left(G, K_{2}\right)=|V(G)|$. As a first non-trivial Ramsey number, let us prove that $R(3,3)=6$.

Proposition 1.1.2. $R(3,3)=6$.

Proof. $R(3,3) \geq 6$ : We have to demonstrate the existence of a red-blue graph on 5 vertices with no monochromatic 3-clique. This is done in Figure 1.1.1. 


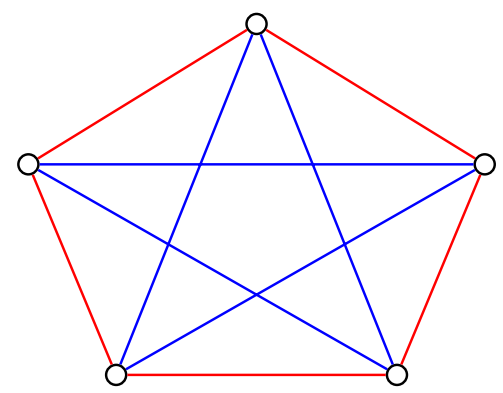

Figure 1.1.1: $R(3,3) \geq 6$.

$R(3,3) \leq 6$ : We have to show that every red-blue graph on 6 vertices contains a monochromatic 3 -clique. Let the vertices be $v, a, b, c, d$, and $e$. We may assume that $v a, v b$, and $v c$ are red. If $a b, a c$, or $b c$ is red, then we have a red 3-clique; otherwise we have a blue 3-clique.

To compute Ramsey numbers is, in general, a very hard problem. For instance, the exact value of $R(i, j)$, where $i, j \geq 3$, is known only when $i=3$ and $j \leq 9$, and for $(i, j) \in\{(4,4),(4,5)\}$. When $G_{1}$ and $G_{2}$ are not complete graphs, more is known. Most important to us is that the cycle versus cycle case has been completely solved. This was done independently by Rosta [15] and by Faudree and Schelp [4] in the 1970s. The formula given here is due to Schwenk (see Harary [6]).

Theorem 1.1.3. Let $n \geq k \geq 3$ and define $f(x, y)=(2 x-1)(y-2[y / 2])$. Then

$$
R\left(C_{n}, C_{k}\right)=\max (6, n+k / 2-1, f(k, n), f(n, k)) .
$$

Another problem in Ramsey theory is to describe $\left(G_{1}, G_{2}\right)$-avoiding graphs. These are graphs that contain neither a red copy of $G_{1}$ nor a blue copy of $G_{2}$. Of particular interest is the characterisation of $\left(G_{1}, G_{2}\right)$-critical graphs, i.e., $\left(G_{1}, G_{2}\right)$-avoiding graphs on $R\left(G_{1}, G_{2}\right)-1$ vertices. For instance, it is well known (and easy to show) that the graph in Figure 1.1.1 is the only (3,3)critical graph (up to isomorphism). As for critical graphs for cycle versus cycle, $\mathrm{Wu}$, Sun, and Radziszowski [18] recently characterised all $\left(C_{n}, C_{4}\right)$-critical graphs for $n \geq 6$.

For more known values and bounds of Ramsey numbers, as well as more on critical graphs, the reader is referred to Radziszowski [12].

There are many generalisations of Ramsey numbers, e.g., colouring complete bipartite graphs or colouring complete graphs with more than two 
colours. In this thesis, we consider a generalisation that was first introduced by Erdős, Faudree, Rousseau, and Schelp [3, 5] in the 1970s:

Definition 1.1.4. The generalised Ramsey number $R\left(\mathcal{G}_{1}, \mathcal{G}_{2}\right)$ is the least positive integer $n$, such that each red-blue graph on $n$ vertices contains a red subgraph from $\mathcal{G}_{1}$ or a blue subgraph from $\mathcal{G}_{2}$.

Observe that when $\left|\mathcal{G}_{1}\right|=\left|\mathcal{G}_{2}\right|=1$, the generalised Ramsey number $R\left(\mathcal{G}_{1}, \mathcal{G}_{2}\right)$ reduces to the ordinary Ramsey number $R\left(G_{1}, G_{2}\right)$. It follows from the definition that $R\left(\mathcal{G}_{1}, \mathcal{G}_{2}\right) \leq R\left(\mathcal{H}_{1}, \mathcal{H}_{2}\right)$ if each $\mathcal{H}_{i}$ is a non-empty subset of $\mathcal{G}_{i}$. Thus

$$
R\left(\mathcal{G}_{1}, \mathcal{G}_{2}\right) \leq R\left(G_{1}, G_{2}\right)
$$

if each $G_{i} \in \mathcal{G}_{i}$. In particular, $R\left(\mathcal{G}_{1}, \mathcal{G}_{2}\right)$ always exists. As well as for ordinary Ramsey numbers, $R\left(\mathcal{G}_{1}, \mathcal{G}_{2}\right)=R\left(\mathcal{G}_{2}, \mathcal{G}_{1}\right)$.

The notions of $\left(\mathcal{G}_{1}, \mathcal{G}_{2}\right)$-avoiding and $\left(\mathcal{G}_{1}, \mathcal{G}_{2}\right)$-critical graphs are completely analogous to the situation when $\left|\mathcal{G}_{1}\right|=\left|\mathcal{G}_{2}\right|=1$.

\subsection{The Bruhat order and poset topology}

Our goal of this section is to define Coxeter groups and the Bruhat order, as well as to give a very brief introduction to poset topology, in particular, the concept of shellability. Before any of this, however, we present some of the basic definitions about posets, in particular, that of a graded poset.

The definitions in Subsection 1.2.1 can be found in, e.g., [16, Chapter 3]. The results in Subsections 1.2.2 and 1.2.3 are all standard; general references are [1, Chapter 2] and [17, Chapter 3], respectively.

\subsubsection{Posets}

A poset (short for partially ordered set) is a set $P$ together with a binary relation $\leq$ on $P$ that is reflexive, antisymmetric, and transitive. Three examples of posets are the integers with the usual $\leq$ (a totally ordered set), the boolean poset $B_{n}$, which is the set of subsets of $[n]$ ordered by inclusion, and the divisibility poset $D_{n}$, which is the set of positive divisors of $n$ ordered by divisibility. In the next subsection, we shall see how the symmetric group, and more generally, any Coxeter group, can be turned into a poset.

In a poset $P$, we say that $y$ covers $x$ if $x<y$ and there is no $z \in P$ such that $x<z<y$; we use the notation $x \triangleleft y$. Furthermore, a chain is a totally ordered subset, and a chain $C$ is saturated if there is no $z \in P-C$ such that $C \cup\{z\}$ is a chain and $x<z<y$ for some $x, y \in C$, and maximal if it is not 
contained in a larger chain. A finite chain $x_{0}<x_{1}<\cdots<x_{k}$ is saturated precisely when $x_{i-1} \triangleleft x_{i}$ for all $i \in[k]$. An $x$-y-chain is a saturated chain from $x$ to $y$. We call $P$ bounded if it has a minimum (denoted by $\hat{0}$ ) and a maximum (denoted by $\hat{1}$ ), which means that $\hat{0} \leq x$ and $\hat{1} \geq x$ for all $x \in P$. An element $x \in P$ is minimal if there is no $y \in P$ with $y<x$, and maximal if there is no $y \in P$ with $y>x$. Observe that a minimal (maximal) element need not be a minimum (maximum).

The notion of a graded poset is the most important one of this subsection. There are a few different, although very similar, definitions. We use the following one:

Definition 1.2.1. A poset $P$ is graded of rank $n$ if every maximal chain consists of $n+1$ elements. In this case, there is a unique rank function $\rho: P \rightarrow\{0,1, \ldots, n\}$ such that $\rho(x)=0$ if $x$ is a minimal element of $P$, and $\rho(y)=\rho(x)+1$ if $x \triangleleft y$ in $P$; $x$ has rank $i$ if $\rho(x)=i$.

\subsubsection{Coxeter groups}

Let $S$ be a set. A Coxeter matrix is a function $m: S \times S \rightarrow\{1,2, \ldots, \infty\}$ such that $m\left(s, s^{\prime}\right)=m\left(s^{\prime}, s\right)$ for all $s, s^{\prime} \in S$, and $m\left(s, s^{\prime}\right)=1 \Leftrightarrow s=s^{\prime}$. Equivalently, $m$ can be represented by a Coxeter graph whose vertex set is $S$ and where $s s^{\prime}$ is an edge if $m\left(s, s^{\prime}\right) \geq 3$, labelled by this number if it is greater than 3. Every Coxeter matrix $m$ determines a group $W$ with the presentation whose set of generators is $S$ and whose relations are $\left(s s^{\prime}\right)^{m\left(s, s^{\prime}\right)}=e$ whenever $m\left(s, s^{\prime}\right)<\infty$. Since $m(s, s)=1, s^{2}=e$, so the generators are involutions. The pair $(W, S)$ is called a Coxeter system and $W$ is called a Coxeter group. The system is irreducible if its Coxeter graph is connected.

Consider now the symmetric group $S_{n}$, i.e., the set of permutations of $[n]$. For each $i \in[n-1]$, let $s_{i}$ denote the adjacent transposition $(i, i+1)$, and let $S$ be the set of all $s_{i}$. For any permutation $\pi=\pi(1) \cdots \pi(i) \pi(i+1) \cdots \pi(n)$ (one line notation), $\pi s_{i}=\pi(1) \cdots \pi(i+1) \pi(i) \cdots \pi(n)$. Hence, $S$ generates $S_{n}$. Furthermore, $s_{i} s_{j}=s_{j} s_{i}$ if $|i-j| \geq 2$ and $s_{i} s_{j} s_{i}=s_{j} s_{i} s_{j}$ if $|i-j|=1$. These facts make it conceivable that $\left(S_{n}, S\right)$ is the Coxeter system whose Coxeter graph is the path

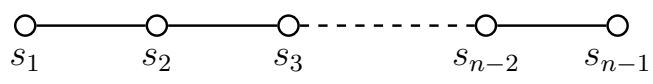

This also turns out to be the case (see, e.g., [1, Proposition 1.5.4]). Hence, the symmetric group is an example of a Coxeter group. 


\section{The Bruhat order}

Let $(W, S)$ be a a Coxeter system. If $w \in W$ is written as a product $w=s_{1} \cdots s_{k}$ of generators, and $w$ cannot be written as a product of fewer generators, then $k$ is the length of $w$, and the word $s_{1} \cdots s_{k}$ is a reduced word for $w$. A subword of a word $s_{1} \cdots s_{q}$ is a word of the form $s_{i_{1}} \cdots s_{i_{m}}$, where $1 \leq i_{1}<\cdots<i_{m} \leq q$.

The Bruhat order is a partial order $\leq$ on $W$. It may be defined as follows (see, e.g., [1, Theorem 2.2.2]):

Definition 1.2.2. Let $u, w \in W$. Then $u \leq w$ if and only if every reduced word for $w$ has a subword that is a reduced word for $u$.

There is a simple characterisation of the Bruhat order on $S_{n}$. For $\sigma \in S_{n}$ and $(k, l) \in[n]^{2}$, let $\sigma[k, l]=|\{i \leq k \mid \sigma(i) \geq l\}|$. We have the following (see, e.g., [1, Theorem 2.1.5]):

Theorem 1.2.3. Let $\sigma, \tau \in S_{n}$. Then $\sigma \leq \tau$ if and only if $\sigma[k, l] \leq \tau[k, l]$ for all $(k, l) \in[n]^{2}$.

As an illustration of Theorem 1.2.3, observe that $34251>21435$, whereas $\sigma=34251$ and $\tau=21534$ are incomparable in the Bruhat order (since $\sigma[1,3]>\tau[1,3]$ and $\sigma[3,5]<\tau[3,5])$.

The Bruhat order turns any Coxeter group into a graded poset whose rank function is the length function. The length of a permutation $\sigma \in S_{n}$ is the number of inversions of $\sigma$, where an inversion is a pair $(i, j) \in[n]^{2}$ such that $i<j$ and $\sigma(i)>\sigma(j)$.

We end this subsection by briefly describing a classic motivation for studying the Bruhat order. Denote by $\mathrm{Fl}_{n}$ the set of all sequences $0 \subset V_{1} \subset$ $V_{2} \subset \cdots \subset V_{n-1} \subset \mathbf{C}^{n}$ of subspaces; the sequences are called flags. There is a bijection between $\mathrm{Fl}_{n}$ and $G / B$, where $G=\mathrm{GL}_{n}(\mathbf{C})$ and $B$ is the subgroup of upper triangular matrices. It turns out that $G / B$ is the disjoint union

$$
G / B=\biguplus_{w \in S_{n}} B w B / B,
$$

where we identify a permutation $w \in S_{n}$ with the corresponding permutation matrix. We call $C_{w}=B w B / B$ a Schubert cell (or a Bruhat cell); the word "cell" is due to the fact that one may define a topology on $\mathrm{Fl}_{n}$ under which each $C_{w}$ is a cell (i.e., homeomorphic to an open ball). The topological closure of a Schubert cell $C_{w}$ is called a Schubert variety; we use the notation $\overline{C_{w}}$. It turns out that

$$
\overline{C_{w}}=\bigcup_{v \leq w} C_{v},
$$

where $\leq$ is the Bruhat order on $S_{n}$. Hence, $\overline{C_{u}} \subseteq \overline{C_{w}}$ precisely when $u \leq w$. 


\subsubsection{Shellability}

Let us first define simplicial complexes. Note that we assume these to be finite.

Definition 1.2.4. A d-dimensional geometric simplex (or just a $d$-simplex) $\sigma$ is the convex hull of $d+1$ affinely independent points (called the vertices of $\sigma$ ) in $\mathbf{R}^{n}$ for some $n \geq d$. The convex hull of $k$ vertices of $\sigma$, where $0 \leq k \leq d+1$, is also a simplex, called a face of $\sigma$; its dimension is $k-1$. The maximal faces are called facets.

A geometric simplicial complex (or just a simplicial complex) $\Delta$ is a finite collection of simplices such that (i) if $\tau \in \Delta$ and $\sigma$ is a face of $\tau$, then $\sigma \in \Delta$, and (ii) if $\sigma, \tau \in \Delta$, then $\sigma \cap \tau$ is a face of both $\sigma$ and $\tau$. We call $F$ a face of $\Delta$ if $F$ is a face of some $\sigma \in \Delta$. The dimension of $\Delta$, denoted by $\operatorname{dim} \Delta$, is the maximum of the dimensions of the facets of $\Delta$. We call $\Delta$ pure if every facet has the same dimension. We view $\Delta$ as a topological space, with the subspace topology induced by the usual topology on $\mathbf{R}^{n}$, by identifying $\Delta$ with the union of its simplices.

Every finite poset defines a simplicial complex, as follows:

Definition 1.2.5. Given a finite poset $P$, identify its elements with $|P|$ affinely independent points in $\mathbf{R}^{n}$ for some $n \geq|P|-1$. The order complex of $P$ is the simplicial complex $\Delta(P)$ whose vertices are the affinely independent points in $\mathbf{R}^{n}$, and whose faces are the convex hulls of the points that correspond to each chain of $P$.

Note that the facets of $\Delta(P)$ are the maximal chains of $P$. Hence, $\Delta(P)$ is pure if and only if $P$ is graded.

We shall consider a property that a pure simplicial complex may have, namely, being shellable.

Definition 1.2.6. Given a pure simplicial complex $\Delta$, let $\mathcal{F}=\left\{F_{1}, \ldots, F_{m}\right\}$ be its set of facets. A shelling order of $\Delta$ is a total order on $\mathcal{F}$, say that $F_{1}<\cdots<F_{m}$, such that

$$
F_{k} \cap\left(\bigcup_{i=1}^{k-1} F_{i}\right)
$$

is a pure subcomplex of $\Delta$ of codimension 1 (i.e., of dimension $\operatorname{dim} \Delta-1$ ) for all $k \in[2, m]$. If $\Delta$ has a shelling order, then $\Delta$ is shellable.

A topological consequence of shellability is the following (see, e.g., [17, Theorem 3.1.3]): 
Theorem 1.2.7. If a pure simplicial complex $\Delta$ is shellable, then $\Delta$ is homotopy equivalent to a wedge of spheres of dimension $\operatorname{dim} \Delta$.

We next present a condition on a poset that implies shellability of its order complex. To this end, let $P$ be a finite, bounded, and graded poset. An edge-labelling of $P$ is a function $\lambda:\left\{(x, y) \in P^{2} \mid x \triangleleft y\right\} \rightarrow Q$, where $Q$ is a totally ordered set. If $\lambda$ is an edge-labelling of $P$ and $x_{0} \triangleleft x_{1} \triangleleft \cdots \triangleleft x_{k}$ is a saturated chain, let $\lambda\left(x_{0}, x_{1}, \ldots, x_{k}\right)=\left(\lambda\left(x_{0}, x_{1}\right), \lambda\left(x_{1}, x_{2}\right), \ldots, \lambda\left(x_{k-1}, x_{k}\right)\right)$. The chain is called increasing if $\lambda\left(x_{i-1}, x_{i}\right) \leq \lambda\left(x_{i}, x_{i+1}\right)$ for all $i \in[k-1]$.

Definition 1.2.8. An edge-labelling $\lambda$ of $P$ is an EL-labelling if, for all $x<y$ in $P$, there is exactly one increasing $x$-y-chain, say $x_{0} \triangleleft x_{1} \triangleleft \cdots \triangleleft x_{k}$, and this chain is lexicographically minimal among the $x$-y-chains in $P$ (i.e., if $y_{0} \triangleleft y_{1} \triangleleft \cdots \triangleleft y_{k}$ is any other $x$-y-chain, then $\lambda\left(x_{j-1}, x_{j}\right)<\lambda\left(y_{j-1}, y_{j}\right)$, where $\left.j=\min \left\{i \in[k] \mid \lambda\left(x_{i-1}, x_{i}\right) \neq \lambda\left(y_{i-1}, y_{i}\right)\right\}\right)$. If $P$ has an EL-labelling, $P$ is said to be EL-shellable.

The reason for this terminology is the following result (see, e.g., [17, Theorem 3.2.2]):

Theorem 1.2.9. Let $P$ be a finite, bounded, and graded poset. If $P$ is EL-shellable, then its order complex $\Delta(P)$ is shellable.

\subsubsection{The Bruhat order on involutions}

It is natural to view the set of involutions in a Coxeter group $W$ as a poset, with the order induced by the Bruhat order on $W$. It turns out that this poset is always graded. This was proved in the case of the symmetric group $S_{n}$ by Richardson and Springer [14] in 1990, and in the 2000s by Incitti [9, 11] (when $W$ is finite of type $A, B$, or $D$ ) and by Hultman [7] (for general $W$ ). The corresponding rank functions were also described in the same papers. That the rank function of the set $I_{n}$ of involutions in $S_{n}$ can be expressed as the average of the number of inversions and the number of exceedances was proved by Incitti [10]; an element $i \in[n]$ is an exceedance of $\sigma \in S_{n}$ if $\sigma(i)>i$. In [10], Incitti also proved that $I_{n}$ is EL-shellable.

One may also view certain subsets of $I_{n}$ as posets, again with the order induced by the Bruhat order on $S_{n}$. For instance, Richardson and Springer [14] proved that the set $F_{n}^{0}$ of fixed-point-free involutions is graded. Hultman [8] studied (in the more general setting of twisted identities, which we describe in Paper B) $F_{n}^{0}$ and $F_{n}^{1}$, the set of involutions with exactly one fixed point. It follows that $F_{n}^{0}$ is graded and Hultman conjectured that the same is true for $F_{n}^{1}$. Recently, Can, Cherniavsky, and Twelbeck [2] proved that $F_{n}^{0}$ is EL-shellable. 



\section{Chapter 2}

\section{Summary of papers}

\section{Paper A: Generalised Ramsey numbers for two sets of cycles}

We study the generalised Ramsey numbers of Definition 1.1.4, where $\mathcal{G}_{1}$ and $\mathcal{G}_{2}$ are sets of cycles, which we denote by $\Gamma_{1}$ and $\Gamma_{2}$. As our first main result, we determine, in particular, all generalised Ramsey numbers $R\left(\Gamma_{1}, \Gamma_{2}\right)$ such that $\Gamma_{1}$ or $\Gamma_{2}$ contains a cycle of length at most 6 , or the shortest cycle in each set is even. This generalises Theorem 1.1.3, as well as some other results. An interesting corollary of our result is that $R\left(C_{n}, C_{4}\right)=R\left(\left\{C_{n}\right\},\left\{C_{3}, C_{4}\right\}\right)$ whenever $n \geq 6$; in other words, having both $C_{3}$ and $C_{4}$ in one of the sets, makes no difference from having only $C_{4}$. We also give a conjecture for the value of $R\left(\Gamma_{1}, \Gamma_{2}\right)$, for general $\left(\Gamma_{1}, \Gamma_{2}\right)$.

In the second part of the paper, we prove that many $\left(\Gamma_{1}, \Gamma_{2}\right)$-avoiding graphs have to be bipartite (when one only considers either the red or the blue edges), even if the number of vertices is only about half the Ramsey number. As our second main result, we characterise most $\left(\Gamma_{1}, \Gamma_{2}\right)$-critical graphs such that $\Gamma_{1}$ or $\Gamma_{2}$ contains a cycle of length at most 5 . For length 4 , this extends the result of $\mathrm{Wu}$, Sun, and Radziszowski [18]. As special cases for lengths 3 and 5 , we obtain complete characterisations of all $\left(C_{n}, C_{3}\right)$-critical graphs for $n \geq 5$, and all $\left(C_{n}, C_{5}\right)$-critical graphs for $n \geq 6$.

\section{Paper B: The Bruhat order on conjugation-invariant sets of involutions in the symmetric group}

We study conjugacy classes, and more generally, unions of conjugacy classes, of involutions in the symmetric group $S_{n}$. Since two permutations are conjugate if and only if, for each $i \geq 1$, they have the same number of disjoint cycles of length $i$, two involutions in $S_{n}$ are conjugate precisely when they 
have the same number of fixed points. Denote by $F_{n}^{a}$ the conjugacy class in $S_{n}$ consisting of the involutions with $a$ fixed points, where $a \in\{0,1, \ldots, n\}$, and for $A \subseteq\{0,1, \ldots, n\}$, let

$$
F_{n}^{A}=\bigcup_{a \in A} F_{n}^{a}
$$

We regard $F_{n}^{A}$ as a poset, with the order induced by the Bruhat order on $S_{n}$.

Our main result is a complete characterisation of the sets $A$ for which $F_{n}^{A}$ is graded. Informally, $F_{n}^{A}$ is graded precisely when $A-\{n\}$ is empty or an "interval," which may consist of a single element if it is 0,1 , or $n-2$. Hence, it follows that $F_{n}^{1}$, the set of involutions with exactly one fixed point, is graded, thereby settling the conjecture of Hultman [8] in the affirmative. As steps in the proof, we show that $F_{n}^{A}$ always has a maximum and describe its minimal elements. We also give the rank function of $F_{n}^{A}$ when it exists; it turns out to be very similar to the rank function of $I_{n}$. Finally, we give a short, new proof of the EL-shellability of $F_{n}^{0}$, the set of fixed-point-free involutions, recently proved by Can, Cherniavsky, and Twelbeck [2]. 


\section{Bibliography}

[1] A. Björner and F. Brenti, Combinatorics of Coxeter groups, Graduate Texts in Mathematics, vol. 231, Springer, New York, 2005.

[2] M. B. Can, Y. Cherniavsky, and T. Twelbeck, Lexicographic shellability of the Bruhat-Chevalley order on fixed-point-free involutions, Israel J. Math. 207 (2015), 281-299.

[3] P. Erdős, R. J. Faudree, C. C. Rousseau, and R. H. Schelp, On cyclecomplete graph Ramsey numbers, J. Graph Theory 2 (1978), 53-64.

[4] R. J. Faudree and R. H. Schelp, All Ramsey numbers for cycles in graphs, Discrete Math. 8 (1974), 313-329.

[5] _ Some problems in Ramsey theory, Theory and applications of graphs (Proc. Internat. Conf., Western Mich. Univ., Kalamazoo, Mich., 1976), Lecture Notes in Math., vol. 642, Springer, Berlin, 1978, pp. 500515 .

[6] F. Harary, A survey of generalized Ramsey theory, Graphs and combinatorics (Proc. Capital Conf., George Washington Univ., Washington, D.C., 1973), Lecture Notes in Math., vol. 406, Springer, Berlin, 1974, pp. $10-17$.

[7] A. Hultman, Fixed points of involutive automorphisms of the Bruhat order, Adv. Math. 195 (2005), 283-296.

[8] _ Twisted identities in Coxeter groups, J. Algebraic Combin. 28 (2008), 313-332.

[9] F. Incitti, The Bruhat order on the involutions of the hyperoctahedral group, European J. Combin. 24 (2003), 825-848.

[10] _ The Bruhat order on the involutions of the symmetric group, J. Algebraic Combin. 20 (2004), 243-261. 
[11] Bruhat order on the involutions of classical Weyl groups, Adv. in Appl. Math. 37 (2006), 68-111.

[12] S. P. Radziszowski, Small Ramsey numbers, Electron. J. Combin. Dynamic Survey 1 (2014), 94 pp. (electronic).

[13] F. P. Ramsey, On a problem of formal logic, Proc. London Math. Soc. 30 (1930), 264-286.

[14] R. W. Richardson and T. A. Springer, The Bruhat order on symmetric varieties, Geom. Dedicata 35 (1990), 389-436.

[15] V. Rosta, On a Ramsey-type problem of J. A. Bondy and P. Erdös. I $\&$ II, J. Combin. Theory Ser. B 15 (1973), 94-120.

[16] R. P. Stanley, Enumerative combinatorics. Vol. 1, Cambridge Studies in Advanced Mathematics, vol. 49, Cambridge University Press, Cambridge, 1997.

[17] M. L. Wachs, Poset topology: tools and applications, Geometric combinatorics, IAS/Park City Math. Ser., vol. 13, Amer. Math. Soc., Providence, RI, 2007, pp. 497-615.

[18] Y. Wu, Y. Sun, and S. P. Radziszowski, Wheel and star-critical Ramsey numbers for quadrilateral, Discrete Appl. Math. 186 (2015), 260-271. 


\section{Part II}

\section{Papers}





\section{Papers}

The articles associated with this thesis have been removed for copyright reasons. For more details about these see:

http://urn.kb.se/resolve?urn=urn:nbn:se:liu:diva-123044 